\title{
Influence of Chronic Cardiovascular Diseases on the Severity of Imported Falciparum Malaria
}

\author{
Bodo Hoffmeister ${ }^{1 *}$, Abner Daniel Aguilar Valdez ${ }^{2}$ \\ 'Department of Respiratory Medicine, Clinic-Group Ernst von Bergmann, Potsdam and Bad Belzig, Niemegker Straße 45, 14806 Bad Belzig, Germany \\ 'Department of Endocrinology, Clinic Group Ernst von Bergmann, Potsdam and Bad Belzig, Niemegker Straße 45, 14806 Bad Belzig, Germany
}

\section{Article Info}

\section{Article Notes}

Received: January 27, 2020

Accepted: February 17, 2020

\section{*Correspondence:}

Dr. Bodo Hoffmeister, Department of Respiratory Medicine, Clinic-Group Ernst von Bergmann, Potsdam and Bad Belzig Niemegker Straße 45, 14806 Bad Belzig, Germany; Email: bodohoff@gmx.de.

(c) 2020 Hoffmeister B. This article is distributed under the terms of the Creative Commons Attribution 4.0 International License.

\section{Keywords:}

Imported falciparum malaria

Risk factors

Aging

Travel-associated diseases

Hypertension

Cardiovascular diseases

Diabetes

Microangiopathy

\section{Abstract}

Falciparum malaria has a unique and complex pathophysiology. While sequestration of parasitized and non-parasitized erythrocytes leads to a progressive obstruction of the microcirculation, a marked systemic inflammation with endothelial dysfunction and consecutive increase in vascular permeability develops. Furthermore, most patients with severe disease present with some degree of hypovolemia. Due to the reduction in pre-load, direct myocardial suppression and increase in after-load acute falciparum malaria exerts diverse effects on the cardiovascular system. Increasing numbers of aging tourists and immigrants with chronic co-morbidities travel to countries where falciparum malaria is endemic. Age has consistently been described as a prominent risk factor for both severe disease and death from imported falciparum malaria in several large studies. Although for long suspected age-related chronic disorders have only recently been identified as risk factors for severe disease. Herein, we review the current concepts of the impact of age-related chronic medical conditions on the severity of imported falciparum malaria.

\section{Introduction}

Malaria is a disease with a unique and complex pathophysiology. Parasitized, as well as non-parasitized red blood cells (RBC) become less deformable and adhere to the endothelium ${ }^{1}$. A progressive obstruction of the microcirculation is the consequence. Simultaneously, a marked systemic inflammation with endothelial dysfunction and increased vascular permeability ensues ${ }^{2}$. In falciparum malaria, the parasite biomass increases 6- to 20-fold with each replication cycle. The exponential growth of parasite biomass every 48 hours is a major reason for the well-described spectrum of life-threatening complications that can occur abruptly and unpredictably within hours of presentation, making Plasmodium falciparum the most dangerous of the five species known to affect humans. Prognostically, the two most important of these so-called criteria of severe malaria (Table 1) are metabolic acidosis and cerebral malaria ${ }^{3}$.

Currently, more than 90 countries and territories are areas at risk of malaria transmission. In countries where malaria is not endemic, it frequently represents the most important imported tropical disease. As such, malaria occurs in specific populations. International travel to countries where malaria is endemic increases steadily. Among such travelers are not only immigrants from endemic countries visiting friends and relatives (VFR), but also increasing numbers of aging tourists with chronic co-morbidities ${ }^{4}$. In addition, patterns of migration change rapidly. Coinciding with an influx of large numbers 
Table 1: Criteria of Severe Malaria in Adults According to the 2014 World Health Organization (WHO) definition

\begin{tabular}{|l|l|}
\hline \multicolumn{1}{|c|}{ Criterion } & \multicolumn{1}{c|}{ Specification } \\
\hline Impaired consciousness & Glasgow coma scale $(\mathrm{GCS})<11$ \\
\hline Metabolic acidosis & A base deficit $>8 \mathrm{mmol} / \mathrm{L}, \mathrm{plasma}$ bicarbonate $<15 \mathrm{mmol} / \mathrm{L}$ or venous lactate $\geq 45 \mathrm{mg} / \mathrm{dL}(>5 \mathrm{mmol} / \mathrm{L})$ \\
\hline Hypoglycaemia & Blood glucose level $<40 \mathrm{mg} / \mathrm{dl}(<2.2 \mu \mathrm{mol} / \mathrm{L})$ \\
\hline Severe malarial anaemia & $\begin{array}{l}\text { Haemoglobin level }<7 \mathrm{~g} / \mathrm{dL} \text { or haematocrit }<20 \% \text { together with a parasite count }>10.000 / \mu \mathrm{L} \text { and } \mathrm{not} \text { related to } \\
\text { other causes than malaria }\end{array}$ \\
\hline Renal impairment & Plasma or serum creatinine $>3 \mathrm{mg} / \mathrm{dL}(>265 \mu \mathrm{mol} / \mathrm{L}$ ) or blood urea $>120 \mathrm{mg} / \mathrm{dL}(>20 \mu \mathrm{mol} / \mathrm{L})$ \\
\hline Jaundice & Bilirubin $>3 \mathrm{mg} / \mathrm{dL}(>50 \mu \mathrm{mol} / \mathrm{L})$ together with a parasite count $>100.000 / \mu \mathrm{L}$ \\
\hline Pulmonary oedema & Confirmed radiologically, or oxygen saturation $<92 \%$ on room air with a respiratory rate $\geq 30 \mathrm{breaths} / \mathrm{min}$ \\
\hline Significant bleeding & Including recurrent or prolonged bleeding from the nose, gums, venepuncture sites, hematemesis or melaena \\
\hline Decompensated shock & Systolic blood pressure $<80 \mathrm{~mm}$ Hg with evidence of impaired perfusion \\
\hline Hyperparasitaemia & P. falciparum parasitemia $>10 \%$; (in non-endemic areas $>5 \%)$ \\
\hline
\end{tabular}

of refugees, for instance, a sharp increase in malaria cases was notified in Germany in $2014^{5}$.

Risk factors for both, severe disease and death, have been identified for imported infections in several studies comprising several thousand cases each $\left({ }^{6-8}\right.$, reviewed in $\left.{ }^{9}\right)$. Among these are inappropriate or no chemoprophylaxis, delay in seeking care and establishment of the diagnosis, the immune status concerning malaria, and co-infection with HIV. Increasing age has also consistently been described as a prominent risk factor in these studies ${ }^{6,10}$. In a large British study, patients aged $>65$ years had 10 times the risk of dying compared to those 18-35 years old ${ }^{6}$. The exact reasons for this association, however, remain to be elucidated.

Here, we review the current concepts of the impact of age-related chronic medical conditions in general, and cardiovascular diseases in particular, on the severity of imported falciparum malaria.

\section{Basic Pathophysiologic Concepts of Falciparum Malaria}

Although still incompletely understood recent research has revealed that four elements play a key role in the pathophysiology of falciparum malaria (Figure 1). Parasitized as well as uninfected red blood cells (RBC) become less deformable and are cleared from the circulation $^{1,11}$. Hemolysis reduces the hemoglobin $(\mathrm{Hb})$ level and may ultimately result in anemia ${ }^{12}$. Cell-free $\mathrm{Hb}$ released from ruptured erythrocytes acts as a nitric oxide (NO) scavenger ${ }^{13}$ and increases the plasma concentration of asymmetric dimethylarginine (ADMA), a NO synthase inhibitor $^{14}$. Reduced NO bioavailability leads not only to increased myocardial wall stress and pulmonary pressures ${ }^{15}$ but also to enhanced endothelial dysfunction with subsequently impaired vasodilation ${ }^{13}$. Reduced RBC deformability also promotes microvascular obstruction ${ }^{1,16}$, the extent of which correlates with metabolic acidosis ${ }^{16}$ and cerebral malaria ${ }^{17}$, the most important prognostic markers in severe malaria. Simultaneously, parasite toxins such as $P$. falciparum histidine-rich protein-2 (PfHRP-2) systemically activate endothelial cells leading to the release of mediators such as Angiopoietin-2 (Ang-2) and von-Willebrand factor (vWF). Angiopoietin-2 promotes the breakdown of endothelial barrier integrity and thus increased vascular permeability, leading to cerebral, peripheral and pulmonary oedema formation with subsequently impaired oxygenation $^{2}$. Finally, due to fluid losses from fever, perspiration, vomiting, diarrhea, insufficient oral fluid intake, increased vascular permeability and other reasons nearly all patients with severe malaria present with some degree of hypovolemia ${ }^{18,19}$.

\section{Effects of Falciparum Malaria on the Cardiovascular System}

The diverse effects of acute falciparum malaria on the cardiovascular system have been described in numerous studies in recent years (reviewed $i^{20}$ ). Hypovolemia and anemia result in reduced pre-load, which is physiologically compensated for by an increase in heart rate and vasoconstriction. More severe tachycardia, lower stroke volume (SV) index, and higher vena cava collapsibility index have been identified in acidotic malaria patients compared to less severely affected individuals ${ }^{21}$. An increase in the systemic vascular resistance (SVR) due to microcirculatory obstruction, vasoconstriction, and other reasons may also contribute to decreases in stroke volume. Higher baseline SVR indexes have been reported in patients who died from severe malaria compared to survivors ${ }^{19}$. Pro-inflammatory cytokines such as tumor necrosis factor (TNF), which are excessively liberated in the context of severe malaria, can directly depress myocardial function ${ }^{22,23}$. Elevated levels of N-terminal brain natriuretic peptide (NT-pro-BNP) and heart-type fatty acid-binding protein (H-FABP) have been found in severe but not in uncomplicated falciparum malaria cases $^{23}$. Importantly, these processes occur simultaneously and may augment each other. With decreasing SV and increasing HR the cardiac cycle becomes less efficient and cardiac output (CO) diminishes ${ }^{24}$. Together with anemia and impaired oxygenation reduced oxygen delivery $\left(\mathrm{DO}_{2}\right)$ to peripheral tissues and hence complicated malaria is the consequence. 


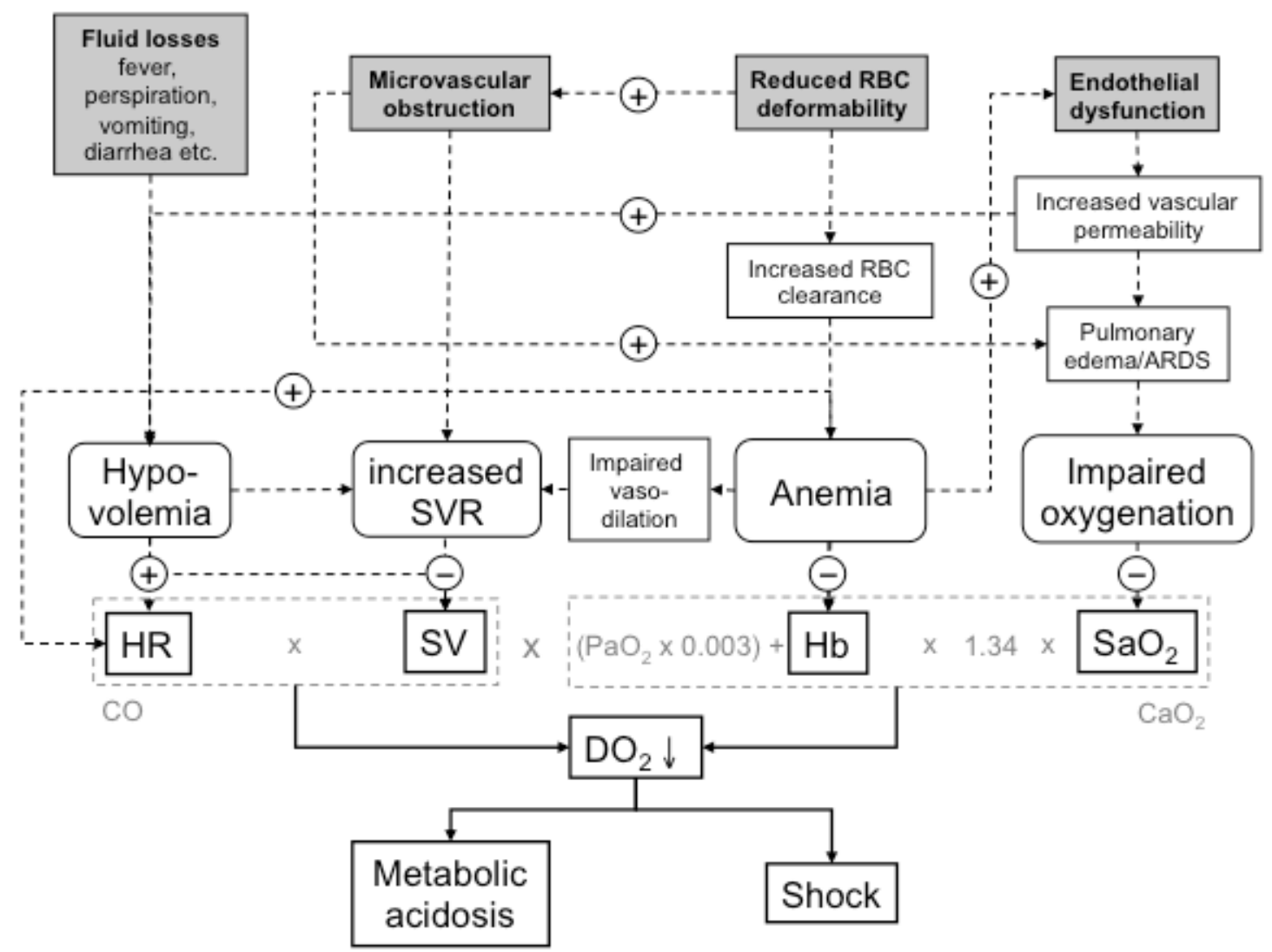

Figure 1: The pathophysiology of falciparum malaria interferes with oxygen delivery to peripheral tissues in several ways. Oxygen delivery $\left(\mathrm{DO}_{2}\right)$ depends on heart rate $(\mathrm{HR})$, stroke volume (SV), hemoglobin level $(\mathrm{Hb})$ and arterial oxygen saturation $\left(\mathrm{SaO}_{2}\right)$. Under physiologic conditions, one parameter can be compensated for by others. However, the four key elements of falciparum malaria pathophysiology (grey boxes) interfere with all components of oxygen delivery simultaneously. The processes may also augment each other. Chronic comorbidities, in particular cardiovascular disorders, may thus facilitate development of life-threatening complications such as metabolic acidosis and shock. This figure has been adapted from ${ }^{34}$. Abbreviations: ARDS: Acute Respiratory Distress Syndrome; $\mathrm{CaO}_{2}$ : Arterial Oxygen Content; $\mathrm{CO}$ : Cardiac Output; $\mathrm{DO}_{2}$ : Oxygen Delivery; Hb: Hemoglobin Level; HR: Heart Rate; $\mathrm{PaO}_{2}:$ Partial Arterial Oxygen Pressure; RBC: Red Blood Cell; $\mathrm{SaO}_{2}$ : Arterial Oxygen Saturation; SV: Stroke Volume; SVR: Systemic Vascular Resistance.

\section{Effects of Chronic Medical Conditions on the Outcome of Falciparum Malaria}

In light of these considerations, it appears plausible that chronic disorders, in particular pre-existing cardiovascular diseases, can add to malaria pathophysiology. In endemic areas, however, the opposite seems to be the case. In fact, as a result of a longstanding host-pathogen interaction malaria has proved a powerful evolutionary driving force for the selection of polymorphisms that confer some degree of innate protection against severe forms of the disease ${ }^{25}$. This selection process focused on the RBC as an essential component of the parasite's life cycle and includes polymorphisms of hemoglobin, intracellular enzymes, channels, surface markers, and morphology of the RBC, to name a few ${ }^{26}$. Since the prevalence of hypertension is strikingly higher in populations constantly exposed to malaria than in those who are not ${ }^{27}$, a co-evolutionary link between these two disorders has been suspected, too $^{28,29}$. Angiotensin II (Ang II) has in fact been shown to inhibit plasmodial growth in vitro directly ${ }^{30}$. It also appears to have a protective effect on the blood-brain barrier integrity ${ }^{31}$. In Indian adults polymorphisms in the angiotensinconverting enzyme (ACE) gene that lead to elevated levels of Ang II and thus hypertension, have been associated with protection against cerebral malaria ${ }^{32}$.

Host genetic factors are estimated to account for one quarter of the total variability in malaria severity ${ }^{32}$. In imported falciparum malaria, the genetic background of the affected populations is highly diverse and hence the clinical situation different from that of endemic areas. Accordingly, chronic co-morbidities may have a significant impact on the severity of imported malaria. Recently, with obesity and diabetes the first two age-related conditions have been identified as risk factors for severe imported falciparum malaria in a nation-wide study from Sweden ${ }^{33}$. Although statistically not significant in the final multivariate model there was also a strong association between hypertension and cardiovascular diseases with an increased risk of severe malaria in this study. Together with dyslipidaemia the latter two conditions were also found to be associated 
with severe disease in patients with falciparum malaria imported to Berlin, Germany ${ }^{34}$. Diabetes, hypertension and lipid disorders play key roles in the development of microangiopathy. Hypertension induces two types of structural changes in the microcirculation: rarefaction and remodelling. Rarefaction describes an abnormal reduction in the density of arterioles, capillaries and possibly venules ${ }^{35}$, while remodelling is a structural reduction in lumen diameter of resistance small arteries and arterioles ${ }^{36}$. Both structural modifications appear in hypertensive individuals, but remodelling seems to play a paramount role in long-term elevation of SVR. Metabolic diseases appear to influence the pattern of structural changes in the microvasculature. While non-diabetic hypertensives develop inward eutrophic remodelling of the microvasculature consisting of a reduction in lumen diameter without changes in vessel cross sectional area ${ }^{36,37}$, diabetics with and without hypertension undergo media vessel hypertrophy withouta reduction of lumen diameter ${ }^{38}$. In addition to such structural changes, functional changes of these vessels are also induced by hypertension and diabetes. Both disorders cause endothelial dysfunction that leads to a reduced bioavailability of local vasodilators (such as NO and prostacyclin) and increased formation of vasoconstrictors and reactive oxygen species (ROS), balancing the microvasculature towards vasoconstriction. Together, these structural and functional changes of the microvasculature result in increased SVR and reduced oxygen delivery to target organs ${ }^{39}$. Pre-existing microangiopathy may thus potentiate the reduced oxygen supply to vital organs caused by imported falciparum malaria, thereby facilitating severe forms of the disease $\mathrm{e}^{34}$. Furthermore, hypertension-related cardiovascular diseases such as congestive heart failure, ischemic heart disease, and atrial fibrillation may contribute themselves to a decreased cardiac output in the setting of acute infection as outlined in Figure 1. Likewise, the risk for sepsis, a condition that shares certain features with falciparum malaria, has been shown to increase with older age, diabetes, atrial fibrillation, dyslipidemia, hypertension, and increasing total number of chronic medical conditions ${ }^{40}$.

\section{Why is That Important?}

The single most important therapeutic intervention in severe malaria is the immediate institution of a causative therapy. Much depends on the timely initiation and correct choice of the antimalarial $^{41}$. However, this is not the only intervention of importance. Optimal supportive care meeting the specific pathophysiologic requirements of the disease is also essential. A recent British study reported a strong, inverse correlation between mortality and the number of cases seen in a given region ${ }^{6}$. In the largest study on severe imported malaria carried out so far a total of 400 patients treated on 45 different intensive care units in France were included ${ }^{42}$. The mortality in this study was $10.5 \%$. When treatment takes place in specialized centers only mortality can be kept below $2 \%{ }^{34,43}$.

The purpose of this mini-review is therefore not only to summarize the diverse effects of acute falciparum malaria on the cardiovascular system. Another main objective is to outline the numerous interventions that exist to support it. Due to simultaneous microcirculatory obstruction with often markedly increased vascular permeability hypovolaemia should be corrected cautiously ${ }^{18,19}$. In case of atrial fibrillation, controlling tachycardia may improve cardiac output, especially in patients with hypertensive heart disease and concomitant congestive heart failure. Adequate hemoglobin levels are important to maintain sufficient oxygenation. This is of particular importance in patients with coronary artery disease. In myocardial failure inotropes can be provided. Early application of positive end-expiratory pressures may not only be beneficial for pulmonary edema of multifactorial etiology, but may also decrease left ventricular afterload, thereby improving left ventricular ejection, cardiac output and myocardial oxygen demand ${ }^{44}$. Significantly elevated blood pressures in hypertensive individuals may be treated with appropriate antihypertensives. Angiotensin-I antagonists are probably superior to other substances in this context ${ }^{28}$. It must be emphasized, however, that unlike the choice of antimalarial none of the aforementioned interventions has been subjected to a controlled randomized clinical trial.

\section{Conclusions}

With its four key elements hypovolemia, increase in systemic vascular resistance, anemia and impaired oxygenation imported falciparum malaria exerts diverse effects on the cardiovascular system. Pre-existing cardiovascular diseases may add to this complex pathophysiology and thereby facilitate the development of complications. Microangiopathy seems to play a pivotal role in this context. Fortunately, the clinician can rely on numerous interventions to optimize supportive care.

\section{References}

1. Dondorp AM, Kager PA, Vreeken J, et al. Abnormal blood flow and red blood cell deformability in severe malaria. Parasitol Today. 2000; 16: 228-32.

2. Yeo TW, Lampah DA, Gitawati R, et al. Angiopoietin-2 is associated with decreased endothelial nitric oxide and poor clinical outcome in severe falciparum malaria. Proc Natl Acad Sci U S A. 2008; 105 : 17097-102.

3. WHO. Guidelines for the treatment of malaria. 3rd ed Vol 19 Suppl I Geneva WHO. 2014; 7-131.

4. Allen N, Bergin C, Kennelly SP. Malaria in the returning older traveler. Trop Dis Travel Med Vaccines. 2016; 2: 2.

5. Vygen-Bonnet $\mathrm{S}$, Stark K. Changes in malaria epidemiology in Germany 2001-2016 a time series analysis. Malar J. 2018; 17: 28.

6. Checkley AM, Smith A, Smith V, et al. Risk factors for mortality from 
imported falciparum malaria in the United Kingdom over 20 years: an observational study. BMJ. 2012; 344: e2116.

7. Krause G, Schoneberg I, Altmann D, et al. Chemoprophylaxis and malaria death rates. Emerg Infect Dis. 2006; 12: 447-51.

8. Legros F, Bouchaud O, Ancelle T, et al. Risk factors for imported fatal Plasmodium falciparum malaria France 1996-2003. Emerg Infect Dis. 2007; 13: 883-8.

9. Luthi B, Schlagenhauf P. Risk factors associated with malaria deaths in travellers: a literature review. Travel Med Infect Dis. 2014; 13: 48-60.

10. Schwartz E, Sadetzki S, Murad H, et al. Age as a risk factor for severe Plasmodium falciparum malaria in nonimmune patients. Clin Infect Dis. 2001; 33: 1774-7.

11. Dondorp AM, Angus BJ, Hardeman MR, et al. Prognostic significance of reduced red blood cell deformability in severe falciparum malaria. Am J Trop Med Hyg. 1997; 57: 507-11.

12. Dondorp AM, Angus BJ, Chotivanich $\mathrm{K}$, et al. Red blood cell deformability as a predictor of anemia in severe falciparum malaria. Am J Trop Med Hyg. 1999; 60: 733-7.

13. Yeo TW, Lampah DA, Tjitra E, et al. Relationship of cell-free hemoglobin to impaired endothelial nitric oxide bioavailability and perfusion in severe falciparum malaria. J Infect Dis. 2009; 200: 1522-9.

14. Yeo TW, Lampah DA, Tjitra E, et al. Increased asymmetric dimethylarginine in severe falciparum malaria: association with impaired nitric oxide bioavailability and fatal outcome. PLoS Pathog. 2010; 6: e1000868.

15. Janka JJ, Koita OA, Traore B, et al. Increased pulmonary pressures and myocardial wall stress in children with severe malaria. J Infect Dis. 2010; 202: 791-800

16. Dondorp AM, Ince C, Charunwatthana P, et al. Direct in vivo assessment of microcirculatory dysfunction in severe falciparum malaria. J Infect Dis. 2008; 197: 79-84.

17. Ponsford MJ, Medana IM, Prapansilp P, et al. Sequestration and microvascular congestion are associated with coma in human cerebral malaria. J Infect Dis. 2011; 205: 663-71.

18. Hanson J, Anstey NM, Bihari D, et al. The fluid management of adults with severe malaria. Crit Care 2015; 18:642.

19. Hanson JP, Lam SW, Mohanty S, et al. Fluid resuscitation of adults with severe falciparum malaria: effects on Acid-base status, renal function, and extravascular lung water. Crit Care Med. 2013; 41: 972-81.

20. Mishra SK, Behera PK, Satpathi S. Cardiac involvement in malaria: an overlooked important complication. J Vector Borne Dis. 2013; 50: 232-5.

21. Yacoub S, Lang HJ, Shebbe M, et al. Cardiac function and hemodynamics in Kenyan children with severe malaria. Crit Care Med. 2010; 38: 940-5.

22. Prabhu SD. Cytokine-induced modulation of cardiac function. Circ Res. 2004; 95: 1140-53.

23. Ehrhardt S, Wichmann D, Hemmer CJ, et al. Circulating concentrations of cardiac proteins in complicated and uncomplicated Plasmodium falciparum malaria. Trop Med Int Health. 2004; 9: 1099-103.

24. Herr J, Mehrfar P, Schmiedel S, et al. Reduced cardiac output in imported Plasmodium falciparum malaria. Malar J. 2011; 10: 160.

25. Evans AG, Wellems TE. Coevolutionary genetics of Plasmodium malaria parasites and their human hosts. Integr Comp Biol. 2002; 42 401-7.

26. Goheen MM, Campino S, Cerami C. The role of the red blood cell in host defence against falciparum malaria: an expanding repertoire of evolutionary alterations. Br J Haematol. 2017; 179: 543-56.

27. Danaei G, Finucane MM, Lin JK, et al. National, regional, and global trends in systolic blood pressure since 1980: systematic analysis of health examination surveys and epidemiological studies with 786 country-years and 5.4 million participants. Lancet. 2011; 377: 56877.

28. Gallego-Delgado J, Walther T, Rodriguez A. The High Blood PressureMalaria Protection Hypothesis. Circ Res. 2016; 119: 1071-5.

29. Eze IC, Bassa FK, Esse C, et al. Epidemiological links between malaria parasitaemia and hypertension: findings from a population-based survey in rural Cote d'Ivoire. J Hypertens. 2019; 37: 1384-92.

30. Saraiva VB, de Souza Silva L, Ferreira-DaSilva CT, et al. Impairment of the Plasmodium falciparum erythrocytic cycle induced by angiotensin peptides. PLoS One. 2011; 6: e17174.

31. Gallego-Delgado J, Basu-Roy U, Ty M, et al. Angiotensin receptors and beta-catenin regulate brain endothelial integrity in malaria. J Clin Invest. 2016; 126: 4016-29.

32. Dhangadamajhi G, Mohapatra BN, Kar SK, et al. Gene polymorphisms in angiotensin I converting enzyme (ACE I/D) and angiotensin Il converting enzyme (ACE2 C-->T) protect against cerebral malaria in Indian adults. Infect Genet Evol. 2010; 10: 337-41.

33. Wyss K, Wangdahl A, Vesterlund M, et al. Obesity and Diabetes as Risk Factors for Severe Plasmodium falciparum Malaria: Results From a Swedish Nationwide Study. Clin Infect Dis. 2017; 65: 949-58.

34. Hoffmeister B, Aguilar Valdez AD. Hypertension is associated with an increased risk for severe imported falciparum malaria: a tertiary care hospital based observational study from Berlin Germany. Malar J. 2019; 18: 410.

35. Feihl F, Liaudet L, Waeber B, et al. Hypertension: a disease of the microcirculation? Hypertension 2006; 48: 1012-7.

36. Park JB, Schiffrin EL. Small artery remodeling is the most prevalent earliest form of target organ damage in mild essential hypertension. J Hypertens. 2001; 19: 921-30.

37. Mathiassen ON, Buus NH, Sihm I, et al. Small artery structure is an independent predictor of cardiovascular events in essential hypertension. J Hypertens. 2007; 25: 1021-6.

38. Schofield I, Malik R, Izzard A, et al. Vascular structural and functional changes in type 2 diabetes mellitus: evidence for the roles of abnormal myogenic responsiveness and dyslipidemia. Circulation. 2002; 106: 3037-43.

39. Nyberg M, Gliemann L, Hellsten Y. Vascular function in health hypertension, and diabetes: effect of physical activity on skeletal muscle microcirculation. Scand J Med Sci Sports. 2015; 25 Suppl 4: 60-73.

40. Wang HE, Shapiro NI, Griffin R, et al. Chronic medical conditions and risk of sepsis. PLoS One. 2012; 7: e48307.

41. Dondorp A, Nosten F, Stepniewska K, et al. Artesunate versus quinine for treatment of severe falciparum malaria a randomised trial. Lancet. 2005 ; 366: 717-25

42. Bruneel F, Tubach F, Corne P, et al. Severe imported falciparum malaria a cohort study in 400 critically ill adults. PLoS One. 2010; 5: e13236.

43. Kurth F, Develoux M, Mechain M, et al. Severe malaria in Europe an 8-year multi-centre observational study. Malar J. 2017; 16: 57.

44. Mahmood SS, Pinsky MR. Heart-lung interactions during mechanical ventilation the basics. Ann Transl Med. 2018; 6: 349 